\title{
GUIDE FOR AUTHORS
}

The Asian Journal of Biodiversity is one of the research journals of Liceo de Cagayan University. The journal is published once a year in January. For paper submission, the paper must be an original copy, about 5,000 words, singlespaced, and with tables, figures and plates. The research abstract must have 200 words and at least 5 keywords or phrases.

\section{Manuscript Preparation}

1. Organize the paper following these major headings: Title, Author(s) and address(es), Abstract, Introduction, Objectives of the Study, Materials and Methods for experimental study or Methodology for non-experimental study, Results, Discussion, Conclusions, and Recommendations (optional).

2. Acknowledgments and Literature Cited. The Literature Cited should substantially consist of articles published in current content-covered or peer-reviewed journals. Minimize citations of unpublished reports and theses.

3. Type the entire manuscript double-spaced on a short white bond paper (8.5x11 in) on one side only with $2.5 \mathrm{~cm}$ margins all around using a Times New Roman font size of 10.

4. References, Acknowledgments, Table Titles, Figures, and Plates. Legends should be typed double-spaced. Number consecutively all pages including title page, Tables, Figures, and Plates.

5. Leave two spaces before and after the major headings and two spaces before and after the sub-headings. Do not use footnotes rather you can use end notes if the discipline needs such.

6. Spell out acronyms or unfamiliar abbreviations when these are mentioned for the first time in the text.

7. Write the scientific names of species completely with author(s) when it is first mentioned in the text and without author in succeeding references. Scientific names should be written in italics or bold face.

8. Do not spell out numbers unless they are used to start a sentence.

9. Use the metric system only or the International System of Units. Use abbreviations of units only beside numerals (e.g. $6 \mathrm{~m}$ ); otherwise, spell 
out the units (e.g. kilometers from here). Do not use plural forms or periods for abbreviations of units. Use the bar for compound units (e.g. $1 \mathrm{~kg} / \mathrm{ha} / \mathrm{yr}$.). Place a zero before the decimal in numbers less than 1 (e.g. $0.25)$

10. When preparing Tables, Figures, and Plates consider the journal's printed page of 5.75 in $x 8.5$ in and the reduction that will be necessary. Titles of tables and captions of Figures and Plates should be as short as possible and understandable without referring to the text. Captions of Figures and Plates should be typed double-spaced in a separate sheet. Figures should consist only of simple line drawings, computer-generated graphics or good quality black and white photographs. Label of Figures and Plates should be written below the image and should be of such a size so that these are still legible even after reducing the size by as much as $50 \%$. Use preferably Adobe Photoshop CS, Adobe InDesign CS and or PDF computer-generated graphics.

11. Cite references in the text as author (year). Writing of et al. in text citation is allowed but complete author names should be indicated in the literature cited on reference list. Cite references in press as (author, in press) and unpublished reference as (author, unpubl. data or author, pers. comm.). If two or more references are cited, arrange them by year.

12. Manuscript should be as concise as the subject and research method permit, generally about 5,000 words or more single-space.

13. To promote anonymous review, authors should not identify themselves directly or indirectly in their papers or in experimental test instruments included in the submission. Single authors should not use the editorial "we".

14. A cover page should show the title of the paper, all authors' names, titles and affiliations, email addresses, and any acknowledgments.

15. The first author or primary author is the person who conducted most of the work described in the paper, and is usually the person who drafted the manuscript. The "senior author" is usually the last person named, and is generally the one who directed or oversaw the project. The names of the "contributing authors" appear between the primary and senior authors, and the order should reflect their relative contribution to the work. 
Pagination: All pages, including tables, figures, plates, appendices and references, should be serially numbered. Major sections should be numbered in Roman numerals. Subsections should not be numbered.

Numbers: Spell out numbers from one to ten, except when used in tables and lists, and when used with mathematical, statistical, scientific, or technical units and quantities, such as distances, weights and measures. Percentage and Decimal Fractions: In nontechnical copy, use the word percent in the text.

Hyphens: Use a hyphen to join unit modifiers or to clarify usage. For example: a cross sectional equation; re-form. See Webster's for correct usage.

Keywords: The abstract must be followed by at least five keywords to assist in indexing the paper and identifying qualified reviewers.

Data Availability: A line immediately following the Keyword identifiers should indicate whether the data are available.

\section{Abstract/ Introduction}

An abstract of about 200 words should be presented on a separate page immediately preceding the text. The Abstract should concisely inform the reader of the manuscript's topic, its methods, and its findings. Keywords and the Data Availability statements should follow the Abstract. The text of the paper should start with a section labeled Introduction," which provides more details about the paper's purpose, motivation, methodology, and findings. Both the Abstract and the Introduction should be relatively nontechnical yet clear enough for an informed reader to understand the manuscript's contribution. The manuscript's title but neither the author's name nor other identification designations, should appear on the Abstract page.

\section{Documentation}

Citations: In-text citations are made using an author-year format. Cited works must correspond to the list of works listed in the "Literature Cited" section.

1. In the text, works are cited as follows: author's last name and year, without comma, in parentheses. 
2. For cited works that include more than one work by an author (or same co -authors) that is published in the same year, the suffix a, b, etc., is to follow the date in the within-text citations and in the "Literature Cited" section.

3. When the author's name is mentioned in the text, it should be listed in the references.

4. Citations to institutional works should use acronyms or short titles where practicable.

5. If the paper refers to statutes, legal treatises, or court cases, citations acceptable in law reviews should be used.

6. All authors should be correctly cited.

\section{Conclusions}

Conclusions should briefly answer the objectives of the study. They are not repetitions of the discussions but are judgments of the results obtained.

\section{Recommendations}

Recommendation is optional, allowed only when results warrant a recommendation.

\section{Literature Cited}

Every manuscript must include a "Literature Cited" section that contains only those works cited within the text. Each entry should contain all information necessary or unambiguous identification of the published work. The style format is based on the Council for Science Editors (CSE) for experimental research and American Psychological Association (APA) for non-experimental research.

\section{Submission of Manuscripts}

Authors should note the following guidelines for submitting manuscripts:

1. Manuscripts currently under consideration by another journal or publisher should not be submitted. The author/s must state upon submission that the work has not been submitted or published elsewhere. The author/s must submit a duly signed Mandatory Copyright Transfer.

2. For manuscripts reporting on field surveys or experiments: If the additional documentation (e.g. questionnaire, case, interview schedule) is sent as a 
separate file, then all information that might identify the authors(s) must be deleted from the instruments.

3. Manuscripts should be submitted via email as Microsoft Word or PDF file to the Editor at email address: rpo.liceo@gmail.com. Please submit separate files for (1) the manuscript's title page with identifying information (not forwarded to reviewers), (2) the manuscript with title page and all other identifying information removed, and (3) any necessary supplement files such as experimental instructions and/or response memorandum on invited revisions. A copy of the research questionnaire or tools is encouraged for submission. The editors and the reviewers need to refer to these tools.

4. Revisions must be submitted within 2 months from the decision letter inviting a revision.

5. Vital information is available at this website: www.asianscientificjournals.com.

\section{Comments}

The Asian Journal of Biodiversity welcomes submission of comments on previous articles. Comments on articles previously published in the Asian Journal of Biodiversity will generally be reviewed by two reviewers, usually an author of the original articles (to assist the editor in evaluating whether the submitted comment represents the prior article accuracy) and an independent reviewer. If a comment is accepted for publication, the original author will be invited to reply. All other editorial requirements, as enumerated above, apply to proposed comments. 